\title{
A 128-BIT MICROPROCESSOR COMPATIBLE PROGRAMMABLE CORRELATOR CHIP FOR USE IN SYNCHRONOUS COMMUNICATION
}

\author{
I. ENIS UNGAN, SATILMIS TOPÇU, and ABDULLAH ATALAR \\ Department of Electrical and Electronics Engineering \\ Bilkent University, 06533 Ankara Turkey
}

\begin{abstract}
A single chip microprocessor compatible 128bit correlator is designed and implemented in $3 \mu \mathrm{m} \mathrm{M} \mathrm{M}^{2} \mathrm{CMOS}$ process. Full-custom design techniques are applied to achieve the best trade off among chip size, speed and power consumption. The chip is to be placed in a microprocessor $(\mu \mathrm{P})$ based tion. The chip is to be placed in a microprocessor $(\mu \mathrm{P})$ based portable data terminal using HF radio communication. It marks the beginning of a synchronous data stream received from word. The sync word can be detected for either inverted or non-inverted input data stream. Two chips can be cascaded to make a 256 -bit correlator. It is fully programmable by a microprocessor to set the number of tolerable errors in detection and to select the bits of the 128-bit (or 256-bit) data stream to be used in the correlation. The latter feature makes the correlator capable for use in detection of distributed sync words and PRBS generation.
\end{abstract}

\section{Introduction}

In digital synchronous data transmission, synchronization (sync) words are used to mark the beginning of the incoming data. Detection of the sync word received from a noisy channel is a difficult problem. In practice, due to the noise in the channel, some of the bits in the data stream may alter and the sync word can be lost if the channel noise is high. An optimum way of detecting the sync word is to use a correlator. The correlator compares the data stream with a predetermined ref erence data and then decides whether the sync word is received or not. Since the channel may be noisy, the correlator must be modified in such a way that it can tolerate some number of errors in the data stream. The bit length of the sync word is a critical parameter for the sync word detection probability, dat integrity and eventually the reliability of the whole communication system. A too short sync-word may cause too many false sync-word detections, whereas a too long one will cause a speed degradation.

A single chip 128-bit correlator is designed to be used in light-weight equipments, such as portable data terminals. It can be used for the sync word detection and PRBS generation. It is $\mu \mathrm{P}$ compatible and hence, it can directly be connected to a $\mu \mathrm{P}$ as a peripheral device. Two chips can be cascaded for 256-bit correlation as well as distributed sync words, and inverted or non-inverted sync words can be detected. The chip is implemented in full-custom because the routing and the placement of the large number of cells can be managed ultimately by the full-custom design. Also, this design style can provide the designer an easy way of testing the chip by reducing the amount of test data. The chip area would be larger if it were implemented in semi-custom design or in gate arrays.

\section{Design of the chip}

The correlator is basically composed of shift, reference mask, status, threshold registers, a comparator, an integrator a decision maker and a controller. Figure 1 shows the block diagram of the correlator. The controller controls all the $\mathrm{I} / \mathrm{O}$ operations and the internal clocking in the chip. The shift register shifts the data from serial data input (SI) to serial data output (SO) and holds the 128 consecutive data bits for correlation. The reference and mask registers are 128-bit write-only registers containing eight 16 -bit shift registers. The reference register is loaded with the sync word and the mask register is loaded with mask data. Logic 1's in the mask register masks the corresponding bits in the 128-bit shift register. The error tolerances for both inverted and non-inverted sync words are stored in the threshold register which is composed of eight 2-bit shift registers. Through the status register, a $\mu \mathrm{P}$ reads the states of
certain internal signals and controls the chip operation for 128 certain internal signals and controls the chip
or 256-bit correlation and PRBS generation.

Correlation starts in the 128-bit comparator which compares the bits in the shift and reference registers and produces a logic 1 for each matched bit. The mask register's logic 1 outputs force the corresponding outputs of the comparator to logic 1 irrespective of the logic levels of shift and reference register contents. The integrator simply counts the matched and masked tents. The integrator simply counts the matched and masked
bits at the output of the comparator and feeds this information to the decision maker. The decision maker uses the integrator output and the error tolerances in the threshold register to decide whether the sync word is detected or not. For 256-bit correlation, two chips are cascaded in a master-slave configuration. The slave chip sends its integrator output to the master chip and the integrator outputs of both chips are added using an 8-bit adder in the master chip.

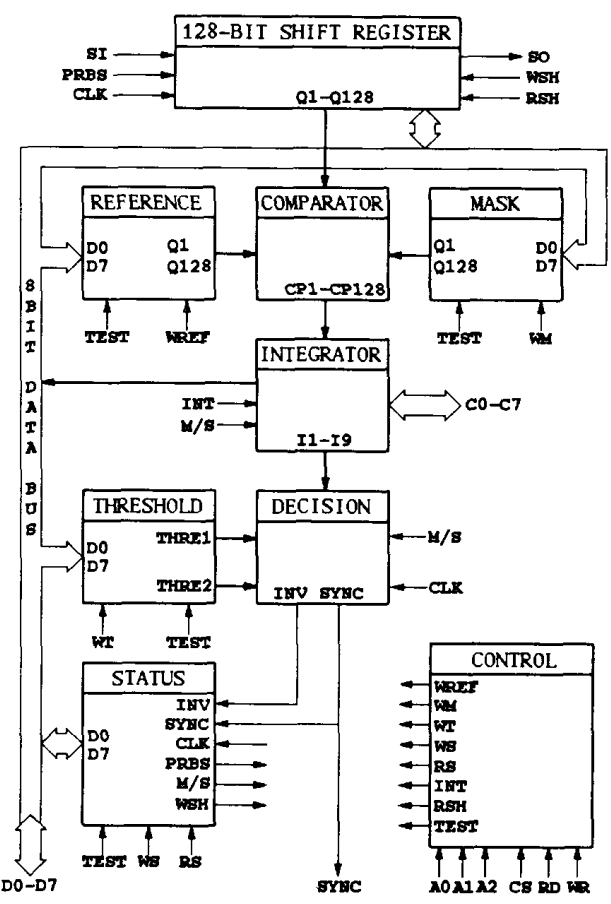

Figure 1: The block diagram of the correlator.

CH.2704-5/89/0000/5146/\$01.00 (C) 1989 IEEE 
A $\mu \mathrm{P}$ can access all the registers in the chip through 8-bit data and 3 -bit address bus. Shift, reference and mask registers are loaded by 8 -bit wide serial data in 16 write cycles. Similarly, the threshold register is loaded in 2 write cycles. The $\mu \mathrm{P}$ can read the most significant 8-bit of the shift register, 8-bit integrator output and three bits of the status register. It can change the reference sync word data, mask data and the threshold data dynamically. The $\mu \mathrm{P}$ is informed on the sync word detection either by an interrupt signal or by polling.

While designing the correlator chip, some "ad hoc design for testability" methods are used; control and observation switching, pin amplification, blocking or degating logic for partitioning [1],[2]. In addition, the scan design technique is applied such that, in the test mode (when test signal is high), the reference, mask, threshold, and status registers are connected in such a way that they form a single shift register which we call as scan path. Since the number of pins available for test purposes is minimal, the number of test pins is increased by multiplex ing of the normal output pins to perform an additional function of acting as test outputs. For blocking or degating, additiona gates are incorporated into the design to inhibit data flow alon the paths, thus partitioning the circuit into smaller modules for the purpose of testing. The test patterns are generated using an automatic test pattern generator by means of path sensitization technique and only stuck-at faults are taken into consideration in relation with the chip layout [2],[3]. The register blocks are instrumental in applying the test inputs to other modules, thus the registers has to be tested first for possible faults before testing could begin on combinational subcircuits. The tests performed on the registers comprise a "flush" and a "shift" test that is, clocking a pattern 001100 , which checks all combinations of initial and next states through the registers, which can be concatenated into a single serial shift register. The comparator and integrator blocks are exhaustively tested with approximately 6000 test vectors by sensitizing independent paths from the layout. Consequently, as a result of the partitioned testing, the number of test patterns are reduced significantly that makes the fault simulation easier and faster. The testability is considerably improved by a $2 \%$ increase in the overall transistor count.

In the layout design of the chip, VLSI complexity is reduced by the construction of a hierarchical layout. The routing of the comparator, shift, reference and mask registers became a serious problem which might not be solved if gate arrays or standard cells were used in the layout design. This routing problem is solved by merging the shift, reference and mask registers and the comparator in a single cell then by constructing an $8 \times 16$ array structure from these cells. The cells in the array are arranged for the loading of the registers so that the array occupies the minimum area. The integrator is constructed from adders th level $(i=0 \ldots 6)$ contains $2^{(7-i)} i$-bit adders. Since these adders are found in a binary tree form, the gate count at the sum and carry stages of each adder is reduced, and a considerable area carry stages of each adder is reduced, and a considerable area
and speed are improved. All primitive cells are designed at the transistor level and complementary static CMOS logic style is used in these cells. In the chip, two-phase clocking is used. Two phase clock is generated and drived by local clock drivers. The clocks are distributed with the metal lines in equal lengths for both phases.

The layout of the chip is drawn and its netlist is extracted by using the Magic layout editor on SUN workstations. The simulations are performed at all levels of the layout hierarchy The Spice and Esim simulators are used for simulating the small sized cells and more complicated cells, respectively. The layout of the chip is plotted in figure 2 .

\section{Chip characteristics}

The 128-bit correlator chip is ready for fabrication in 3 micron double metal CMOS technology. According on the simulation results, the chip can make correlation at $1 \mathrm{Mbps}$ rate with a power dissipation less than $80 \mathrm{~mW}$. The chip character- istics are given in table 1.

\begin{tabular}{|c|c|}
\hline Technology & $3 \mu \mathrm{m}$-well $\mathrm{M}^{2} \mathrm{CMOS}$ \\
\hline Active area & $4 \mathrm{~mm} \times 4.3 \mathrm{~mm}$ \\
\hline Total area & $5.6 \mathrm{~mm} \times 5.2 \mathrm{~mm}$ \\
\hline Device count & 15,000 transistors \\
\hline Maximum frequency & $1 \mathrm{MHz}$ \\
\hline Maximum power & $80 \mathrm{~mW} @ 1 \mathrm{MHz}$ \\
\hline Pin count & 28 \\
\hline
\end{tabular}

Table 1: The Chip characteristics.

\section{Acknowledgement}

N. Aral and T. Ergün of ASELSAN made most of the system design of the correlator. This research was sponsored by NATO's Scientific Affairs Division in the framework of the Science for Stability Programme.

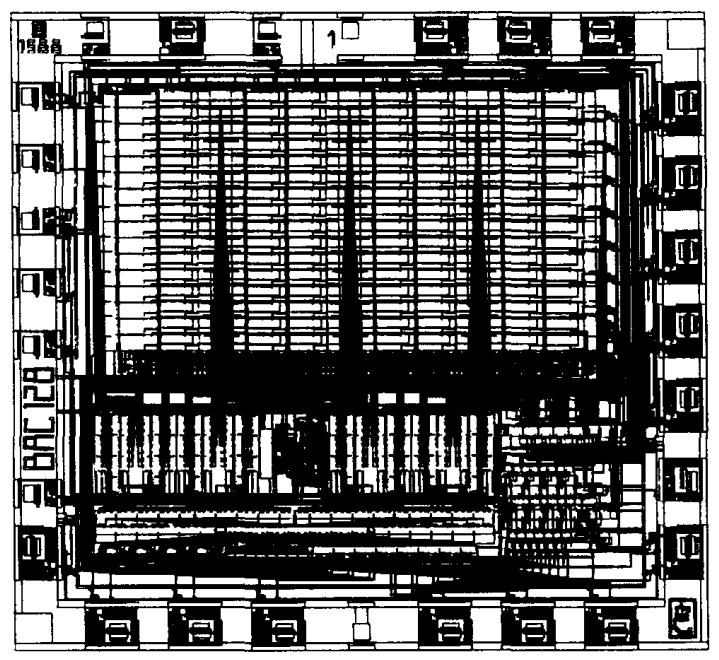

Figure 2: The layout of the correlator.

\section{References}

[1] T. W. Williams and K. P. Parker, "Design For Testability-A Survey", Proc. IEEE, vol. 71, pp. 98-112, Jan. 1983.

[2] G. Russell, D. J. Kinniment, E. G. Chester, M. R. McLauhlan, CAD for VLSI. England, Van Nostrand Reinhold, 1985.

[3] J. A. Abraham and W. K. Fuchs, "Fault and Error Models for VLSI", Proc. IEEE, vol. 74, pp. 639-654, May 1986. 